\title{
Inhaled corticosteroid withdrawal may improve outcomes in elderly patients with COPD exacerbation: a nationwide database study
}

\author{
Taisuke Jo (i] ${ }^{1,2}$, Hideo Yasunaga ${ }^{3}$, Yasuhiro Yamauchi ${ }^{2}$, Akihisa Mitani², \\ Yoshihisa Hiraishi ${ }^{2}$, Wakae Hasegawa ${ }^{2}$, Yukiyo Sakamoto ${ }^{2}$, Hiroki Matsui ${ }^{3}$, \\ Kiyohide Fushimi ${ }^{4}$ and Takahide Nagase ${ }^{2}$
}

Affiliations: ${ }^{1}$ Dept of Health Services Research, Graduate School of Medicine, The University of Tokyo, Tokyo, Japan. ${ }^{2}$ Dept of Respiratory Medicine, Graduate School of Medicine, The University of Tokyo, Tokyo, Japan. ${ }^{3}$ Dept of Clinical Epidemiology and Health Economics, School of Public Health, The University of Tokyo, Tokyo, Japan. ${ }^{4}$ Dept of Health Policy and Informatics, Tokyo Medical and Dental University Graduate School of Medicine, Tokyo, Japan.

Correspondence: Taisuke Jo, Depts of Health Services Research and Respiratory Medicine, Graduate School of Medicine, The University of Tokyo, 7-3-1 Hongo, Bunkyo-ku, Tokyo 113-0033, Japan. E-mail: jo-taisuke@uumin.ac.jp

\section{ABSTRACT}

Background: Inhaled corticosteroids (ICSs) are used for advanced-stage chronic obstructive pulmonary disease (COPD). The application and safety of ICS withdrawal remain controversial.

This study aimed to evaluate the association between ICS withdrawal and outcomes in elderly patients with COPD with or without comorbid bronchial asthma, who were hospitalised for exacerbation.

Patients and methods: We conducted a retrospective cohort study using the Japanese Diagnosis Procedure Combination database from July 2010 to March 2016. We identified patients aged $\geqslant 65$ years who were hospitalised for COPD exacerbation. Re-hospitalisation for COPD exacerbation or death, frequency of antimicrobial medicine prescriptions and frequency of oral corticosteroid prescriptions after discharge were compared between patients with withdrawal and continuation of ICSs using propensity score analyses, namely 1-2 propensity score matching and stabilised inverse probability of treatment weighting.

Results: Among 3735 eligible patients, 971 and 2764 patients had ICS withdrawal and continuation, respectively. The hazard ratios (95\% confidence intervals) of re-hospitalisation for COPD exacerbation or death for ICS withdrawal compared to continuation were $0.65(0.52-0.80)$ in the propensity score matching and $0.71(0.56-0.90)$ in the inverse probability of treatment weighting. The frequency of antimicrobial prescriptions but not corticosteroid prescriptions within 1 year was significantly less in the ICS withdrawal group. Among patients with comorbid bronchial asthma, ICS withdrawal was significantly associated with reduced re-hospitalisation for COPD exacerbation or death only in the propensity score matching analysis. Conclusion: ICS withdrawal after COPD exacerbation was significantly associated with reduced incidences of re-hospitalisation or death among elderly patients, including those with comorbid bronchial asthma.

@ERSpublications

ICS withdrawal after COPD exacerbation is significantly associated with reduced incidences of re-hospitalisation or death among elderly patients. ICS withdrawal may thus be considered as a treatment option in elderly patients with COPD. http://bit.ly/2rMg4wK

Cite this article as: Jo T, Yasunaga $\mathrm{H}$, Yamauchi $\mathrm{Y}$, et al. Inhaled corticosteroid withdrawal may improve outcomes in elderly patients with COPD exacerbation: a nationwide database study. ERJ Open Res 2020; 6: 00246-2019 [https://doi.org/10.1183/23120541.00246-2019].

This article has supplementary material available from openres.ersjournals.com.

Received: 12 Sept 2019 | Accepted after revision: 27 Nov 2019

Copyright @ERS 2020 This article is open access and distributed under the terms of the Creative Commons Attribution Non-Commercial Licence 4.0. 


\section{Introduction}

Inhaled long-acting muscarinic antagonists (LAMAs) and inhaled long-acting $\beta 2$-agonists (LABAs), either alone or in combination, are the first-line treatment for symptomatic chronic obstructive pulmonary disease (COPD). Treatment with long-acting bronchodilators is also effective for preventing COPD exacerbation, which represents acute worsening in patients with COPD [1]. Prevention of COPD exacerbation may in turn improve the prognosis of patients with COPD.

However, the role of inhaled corticosteroids (ICSs) in patients with COPD remains uncertain. Studies of ICSs in combination with bronchodilators in patients with stable COPD showed mixed results in terms of preventing COPD exacerbation [2,3]. Nevertheless, ICSs in addition to bronchodilators are still considered a major therapeutic option in patients who have experienced an exacerbation leading to hospital admission. Moreover, ICS/LABA is recommended as the first-line treatment choice in patients with asthma-COPD overlap (ACO) [4]. However, elderly patients are generally vulnerable to adverse drug reactions from inhalers [5]. Adverse effects including candidiasis, cataract, glaucoma, diabetes, bone fracture, and pneumonia have been associated with high-dose ICSs [6] and have been raised as an issue, particularly in elderly patients [7, 8]. In addition, an increased risk of lower respiratory tract infection associated with the use of ICSs has also been reported in patients with bronchial asthma $[9,10]$.

Most studies that have recently evaluated the impact of ICS withdrawal showed no increase in the risk of re-exacerbation [11-13]. However, one meta-analysis showed no significant reduction in the number of exacerbations after ICS withdrawal, whereas symptoms evaluated with the St. George's Respiratory Questionnaire, change in forced expiratory volume in $1 \mathrm{~s}$, and time to first exacerbation, were better in patients who continued ICSs [14]. Hence the application and safety of ICS withdrawal in patients at high risk of COPD exacerbation remain controversial, particularly among patients with ACO. Moreover, no studies have yet evaluated the impact of ICS withdrawal focusing on elderly patients, despite the higher mortality of COPD in the elderly.

The aim of this study was to determine the effect of withdrawal of ICSs after hospitalisation for COPD exacerbation on clinical outcomes, including re-exacerbation and death, in elderly patients with COPD, including those with ACO.

\section{Patients and methods}

\section{Data source}

This retrospective cohort study analysed inpatient and outpatient data from the Japanese Diagnosis Procedure Combination database [15]. Outpatient data were collected from approximately 250 hospitals across Japan from July 2010 to March 2016. The inpatient database included information on age, sex, body height and weight (body mass index), primary and comorbid diagnoses, Barthel Index scores [16] at admission and discharge, smoking history, Hugh-Jones score, operative procedures and anaesthesia, discharge status, medications and treatments in both inpatient and outpatient settings, dates of admission and discharge, and residential region. Residential regions were categorised by district into five regions from the north east to the south west of Japan: Hokkaido and Tohoku; Kanto; Chubu; Kansai; and Chugoku, Shikoku, Kyushu, and Okinawa. These regions may reflect differences in the natural environment as well as the social environment in Japan [17]. Diagnoses were recorded according to the International Statistical Classification of Diseases and Related Health Problems, 10th revision (ICD-10) codes.

This study was approved by the Institutional Review Board of The University of Tokyo, which waived the requirement for informed patient consent because of the anonymous nature of the data.

\section{Patient selection}

We extracted data for patients aged $\geqslant 65$ years who were hospitalised for COPD exacerbation. We included patients with outpatient prescriptions for ICSs with either LABAs or LAMAs alone or in combination before their hospitalisation for COPD exacerbation. The ICD-10 codes used to identify patients with COPD, COPD exacerbation, and bacterial pneumonia are listed in table S1.

The ICS withdrawal group included patients for whom ICSs were not prescribed during the index hospitalisation or the entire period of post-hospitalisation follow-up. The ICS continuation group (control group) comprised patients for whom ICSs were prescribed during and after the index hospitalisation (figure 1a).

We performed subgroup analyses to evaluate patients with and without comorbid bronchial asthma. Patients with bronchial asthma were defined as those with ICD-10 codes for bronchial asthma (table S1) during the observation period before and during hospitalisation for COPD exacerbation.

We also performed sensitivity analyses in patients with COPD aged $\geqslant 40$ years, and stratified the patients using a cut-off age of 70 years. 


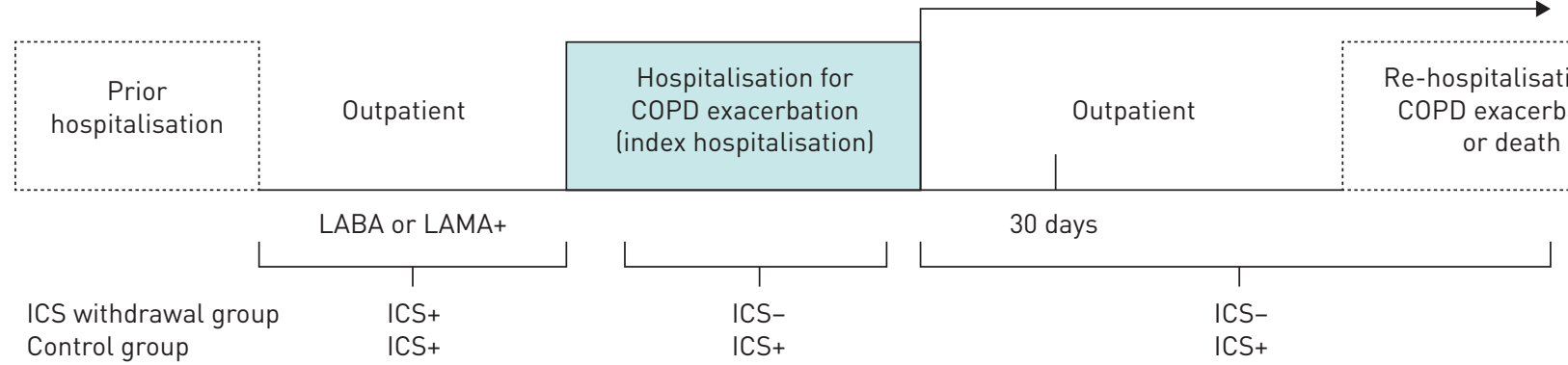

b)

23047 patients who were hospitalised for COPD exacerbation (index hospitalisation) and had outpatient data before and after the index hospitalisation were included

2900 patients aged $<65$ years were excluded

14418 patients without long-acting bronchodilators before index hospitalisation were excluded

5729 patients aged $\geqslant 65$ years who were hospitalised for COPD exacerbation and had ICS with bronchodilators (LABA or LAMA) prescribed before the hospitalisation for COPD exacerbation

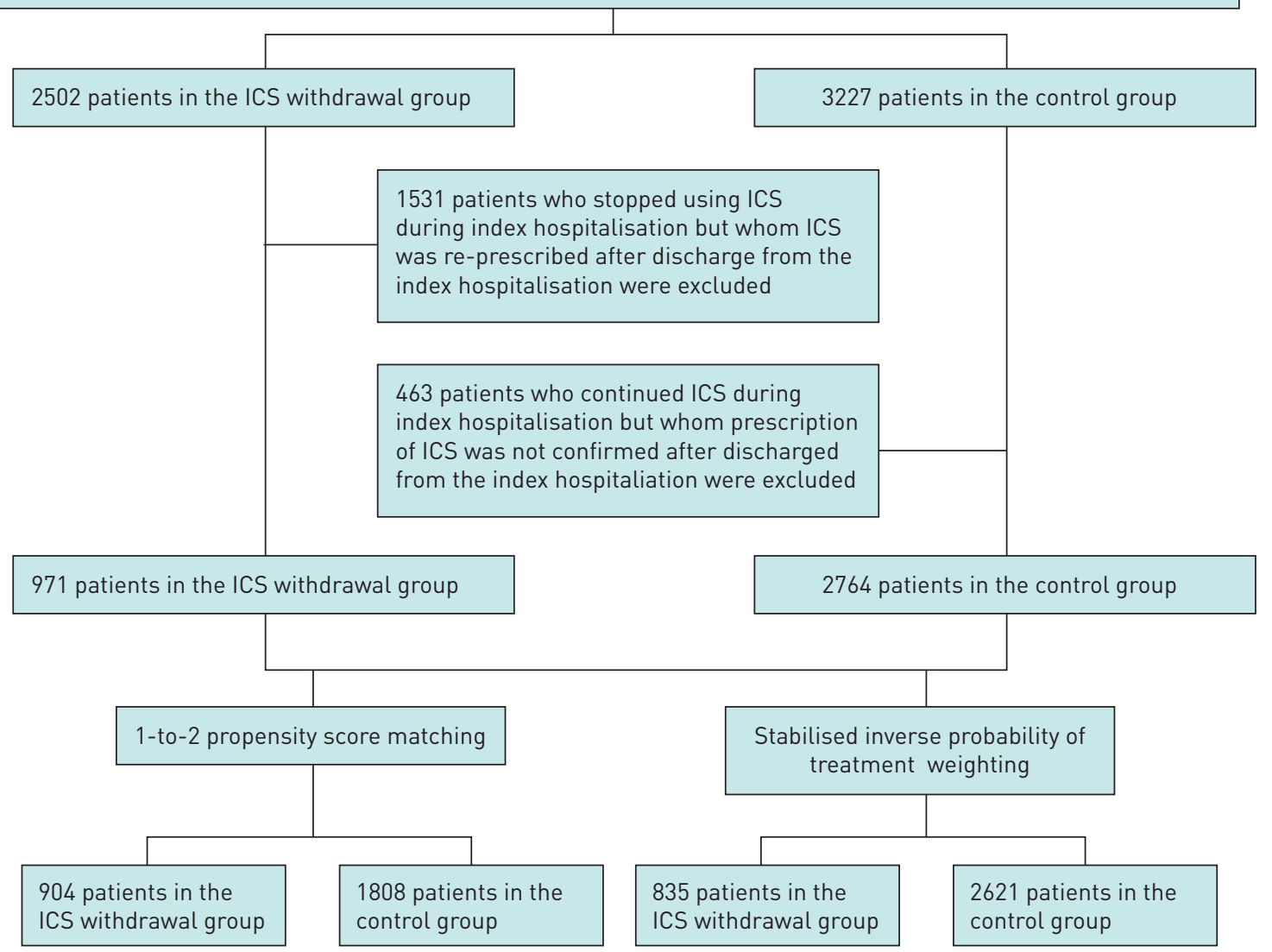

FIGURE 1 Schematic diagram of study groupings. a) Study schematic showing each period evaluated in the study. Variables defining patient characteristics and comorbidities were obtained from the hospitalisation for COPD exacerbation, outpatient data, and data from prior hospitalisations. The outcomes were re-hospitalisation or death and incidences of prescriptions at 30 days and 1 year after the hospitalisation. The ICS withdrawal group was identified by discontinuation of the prescription during and after the hospitalisation for COPD exacerbation. b) Flow diagram of the study patients. LABA: long-acting $\beta_{2}$-agonist; LAMA: long-acting muscarinic antagonist; ICS: inhaled corticosteroid. 


\section{Outcomes}

The main outcome measure was re-hospitalisation for COPD exacerbation or all-cause mortality within 30 days and 1 year after discharge from the index hospitalisation. The index date for patient follow-up was the date of discharge from the index hospitalisation. The secondary outcome measures were prescriptions of antimicrobials and systemic corticosteroids within 30 days and 1 year after discharge from the index hospitalisation.

\section{Statistical analyses}

Propensity score matching (PSM) [18] and propensity-score stabilised inverse probability of treatment weighting (IPTW) [19] were performed to account for the differences in baseline characteristics, including comorbidities and treatments, between the ICS withdrawal and continuation groups. Both methods utilise propensity scores; the former evaluates average treatment effects of the treated, while the latter evaluates average treatment effects. The baseline characteristics, comorbidities (table S1) and treatments before and during hospitalisation for COPD exacerbation assessed were listed in table S2. Medications and treatments for COPD in outpatient settings before the index hospitalisation served as proxies for the severity of COPD.

Continuous variables were reported as mean \pm SD or median and interquartile range (IQR). Nonparametric values were compared using a Mann-Whitney U-test. Dichotomous and categorical variables were reported as frequency and compared using the Chi-squared test. The ICD-10 codes used to identify comorbidities are shown in table S1 and the drugs used in this study are listed in table S2. Anti-influenza medicines were included in antimicrobials.

We estimated propensity scores using a multivariable logistic regression model, including the aforementioned covariates as dependent variables [18]. For PSM, we set a calliper width at $20 \%$ of the SD of the propensity scores, and performed 1-2 matching without replacement. Propensity-score stabilised IPTW employs a pseudo-population in which the treatment is independent of the measured potential confounders, meaning that the weighted treatment effect estimates will be less biased. Stabilised IPTW methods can estimate the average treatment effects over the marginal distribution, while preserving sample size. We evaluated the balance in baseline characteristics between the two groups using the standardised mean difference. Significant imbalance was defined as an absolute standardised difference of $>10 \%$ [20].

Cumulative hazard curves were drawn, and a Cox proportional hazard model was used to estimate the hazard ratios (HR) and 95\% confidence intervals (CIs) of re-hospitalisation for COPD exacerbation or death. The frequencies of antimicrobial and systemic corticosteroid prescriptions in the two groups were compared by Poisson regression analyses. Both Cox and Poisson regression models were adjusted for within-hospital clustering by employing a robust variance estimator (also known as a sandwich variance estimator) [21], which produce unbiased SE estimators for regression coefficients in cluster-correlated data with a large sample setting and sufficient number of events. We included the background characteristics that were significantly imbalanced after 1-2 PSM and stabilised IPTW in both of the regression models.

Data analyses were carried out using SPSS version 23.0 (IBM SPSS Inc., Armonk, NY, USA) and STATA software version 14.1 (StataCorp LP, TX, USA). Values of $\mathrm{p}<0.05$ indicated statistical significance.

\section{Results}

We identified 23047 patients hospitalised for COPD exacerbation. Among these, 3735 patients were aged $\geqslant 65$ years and had both ICS and bronchodilators prescribed before hospitalisation for COPD exacerbation (index hospitalisation). ICSs were withdrawn in 971 of these patients and continued in 2764 patients during and after the index hospitalisation (figure 1b). The proportions of patients with bacterial pneumonia at the index hospitalization in the ICS withdrawal group and control group were $83.5 \%$ and 85.5\% ( $\mathrm{p}=0.093)$ after PSM and 78.3\% and 78.0\% ( $\mathrm{p}=0.914)$ after stabilised IPTW, respectively.

The baseline characteristics of patients in the ICS withdrawal and continuation groups are shown in table 1. A higher proportion of patients in recent years had ICS withdrawal. Body mass index was lower in the ICS withdrawal compared to the ICS continuation group. The frequency of hospitalisation before the index hospitalisation was higher and the observation period was longer in the ICS withdrawal group.

Comorbidities and treatments before and/or during the index hospitalisation are shown in table 2. The proportion of patients with bronchial asthma was lower in the ICS withdrawal group compared to the control group $(36.3 \%$ versus $65.5 \%$ ), whereas the proportion of patients with malignancy and interstitial pneumonia was higher in the ICS withdrawal group. LABAs were prescribed before hospitalisation more frequently in the ICS withdrawal group, whereas LAMAs, theophylline, macrolides, and corticosteroids were prescribed more frequently in the ICS continuation group. Prescription of fixed-dose LABAs/LAMAs in a single inhaler as well as LABAs were prescribed more during hospitalisation in patients with ICS withdrawal, whereas LAMAs were prescribed more in control patients. The distributions of most covariates 
TABLE 1 Baseline characteristics of patients with COPD with and without inhaled corticosteroid (ICS) withdrawal, before and after 1-2 propensity score (PS) matching and after stabilised inverse probability weighting (IPTW)

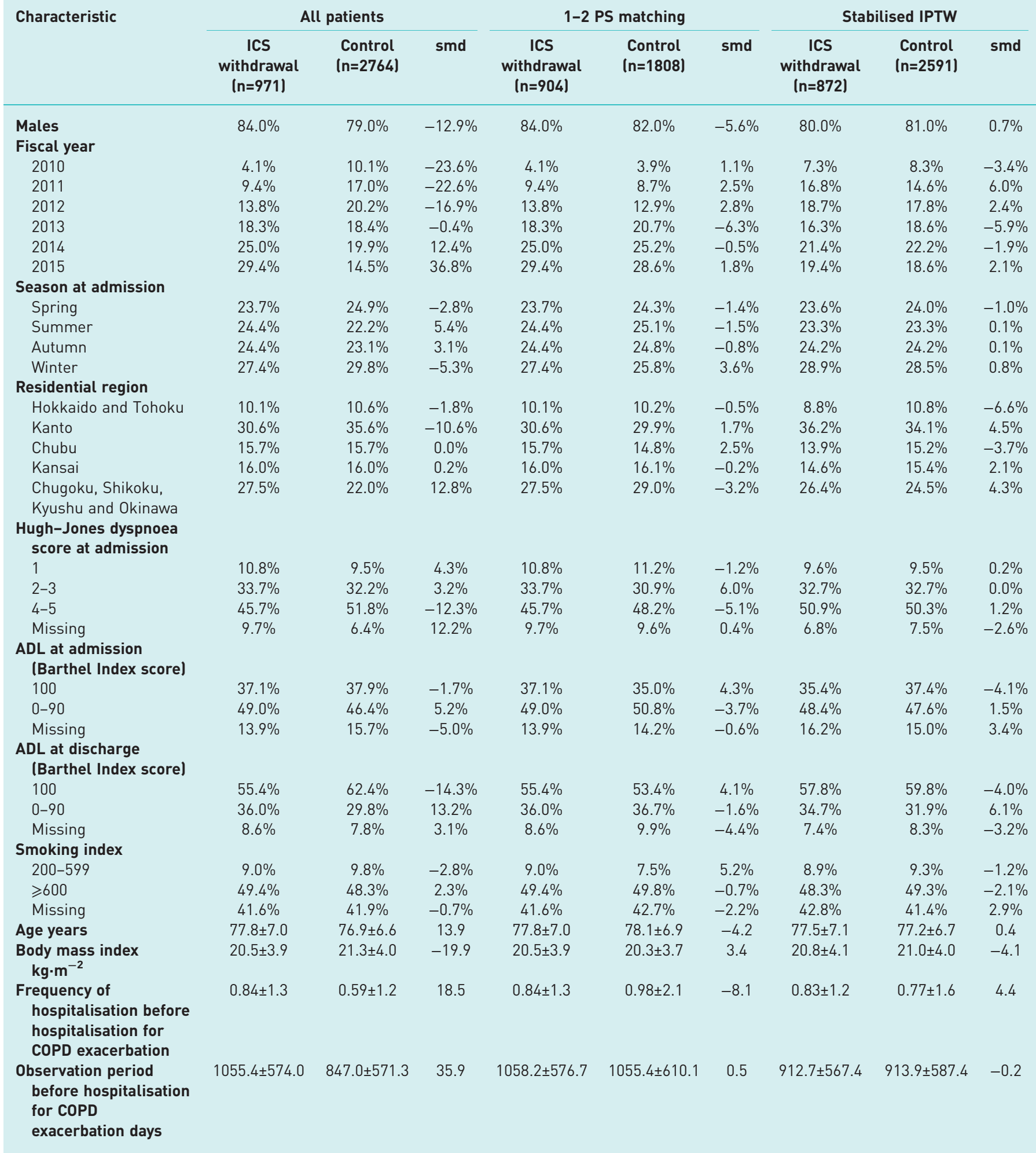

Data are presented as mean \pm SD unless otherwise stated. smd: standardised mean difference; ADL: activities of daily living. 
TABLE 2 Comorbidities during hospitalisation and treatments for COPD with and without inhaled corticosteroid (ICS) withdrawal, before and after 1-2 propensity score (PS) matching and after stabilised inverse probability weighting (IPTW)

\begin{tabular}{|c|c|c|c|c|c|c|c|c|}
\hline \multicolumn{3}{|c|}{ All patients } & \multicolumn{3}{|c|}{ 1-2 PS matching } & \multicolumn{3}{|c|}{ Stabilised IPTW } \\
\hline $\begin{array}{c}\text { ICS } \\
\text { withdrawal } \\
{[\mathrm{n}=971) \times 100}\end{array}$ & $\begin{array}{l}\text { Control } \\
\begin{array}{l}\text { (n=2764) } \\
\times 100\end{array}\end{array}$ & smd & $\begin{array}{c}\text { ICS } \\
\text { withdrawal } \\
(n=904) \times 100\end{array}$ & $\begin{array}{l}\text { Control } \\
\text { [n=1808) } \\
\times 100\end{array}$ & smd & $\begin{array}{c}\text { ICS } \\
\text { withdrawal } \\
(n=872) \times 100\end{array}$ & $\begin{array}{l}\text { Control } \\
\text { (n=2591) } \\
\times 100\end{array}$ & smd \\
\hline $0.14 \%$ & $0.08 \%$ & $16.8 \%$ & $0.14 \%$ & $0.14 \%$ & $-0.2 \%$ & $0.12 \%$ & $0.11 \%$ & $3.9 \%$ \\
\hline $0.10 \%$ & $0.07 \%$ & $12.5 \%$ & $0.10 \%$ & $0.11 \%$ & $-2.2 \%$ & $0.08 \%$ & $0.08 \%$ & $-1.1 \%$ \\
\hline $0.21 \%$ & $0.22 \%$ & $-2.4 \%$ & $0.21 \%$ & $0.22 \%$ & $-3.5 \%$ & $0.27 \%$ & $0.22 \%$ & $11.5 \%$ \\
\hline $0.06 \%$ & $0.07 \%$ & $-2.1 \%$ & $0.06 \%$ & $0.07 \%$ & $-4.0 \%$ & $0.05 \%$ & $0.07 \%$ & $-4.7 \%$ \\
\hline $0.11 \%$ & $0.05 \%$ & $24.5 \%$ & $0.11 \%$ & $0.11 \%$ & $-0.9 \%$ & $0.07 \%$ & $0.07 \%$ & $2.6 \%$ \\
\hline $0.37 \%$ & $0.66 \%$ & $-61.0 \%$ & $0.37 \%$ & $0.39 \%$ & $-4.6 \%$ & $0.58 \%$ & $0.58 \%$ & $-0.2 \%$ \\
\hline $0.24 \%$ & $0.22 \%$ & $4.2 \%$ & $0.24 \%$ & $0.21 \%$ & $7.2 \%$ & $0.22 \%$ & $0.22 \%$ & $-0.6 \%$ \\
\hline $0.04 \%$ & $0.03 \%$ & $3.1 \%$ & $0.04 \%$ & $0.04 \%$ & $-2.6 \%$ & $0.04 \%$ & $0.03 \%$ & $1.6 \%$ \\
\hline $0.00 \%$ & $0.01 \%$ & $3.8 \%$ & $0.00 \%$ & $0.00 \%$ & $4.7 \%$ & $0.01 \%$ & $0.01 \%$ & $-0.2 \%$ \\
\hline $0.02 \%$ & $0.01 \%$ & $10.9 \%$ & $0.02 \%$ & $0.02 \%$ & $2.6 \%$ & $0.01 \%$ & $0.01 \%$ & $-0.5 \%$ \\
\hline $0.02 \%$ & $0.02 \%$ & $0.6 \%$ & $0.02 \%$ & $0.03 \%$ & $-6.5 \%$ & $0.02 \%$ & $0.02 \%$ & $-1.7 \%$ \\
\hline $0.02 \%$ & $0.02 \%$ & $2.2 \%$ & $0.02 \%$ & $0.02 \%$ & $-0.8 \%$ & $0.02 \%$ & $0.02 \%$ & $0.7 \%$ \\
\hline $0.21 \%$ & $0.22 \%$ & $-0.8 \%$ & $0.21 \%$ & $0.20 \%$ & $2.9 \%$ & $0.22 \%$ & $0.22 \%$ & $0.2 \%$ \\
\hline $0.14 \%$ & $0.14 \%$ & $1.8 \%$ & $0.14 \%$ & $0.14 \%$ & $-0.3 \%$ & $0.17 \%$ & $0.14 \%$ & $9.6 \%$ \\
\hline $0.11 \%$ & $0.10 \%$ & $3.8 \%$ & $0.11 \%$ & $0.11 \%$ & $1.1 \%$ & $0.11 \%$ & $0.10 \%$ & $0.5 \%$ \\
\hline $0.04 \%$ & $0.03 \%$ & $7.5 \%$ & $0.04 \%$ & $0.05 \%$ & $5.0 \%$ & $0.03 \%$ & $0.03 \%$ & $1.2 \%$ \\
\hline $0.03 \%$ & $0.02 \%$ & $5.0 \%$ & $0.03 \%$ & $0.03 \%$ & $-0.3 \%$ & $0.02 \%$ & $0.03 \%$ & $-1.5 \%$ \\
\hline $0.03 \%$ & $0.02 \%$ & $5.3 \%$ & $0.03 \%$ & $0.05 \%$ & $-9.2 \%$ & $0.03 \%$ & $0.03 \%$ & $0.7 \%$ \\
\hline $0.04 \%$ & $0.03 \%$ & $5.2 \%$ & $0.04 \%$ & $0.05 \%$ & $-2.4 \%$ & $0.03 \%$ & $0.03 \%$ & $-1.6 \%$ \\
\hline $0.19 \%$ & $0.22 \%$ & $-6.9 \%$ & $0.19 \%$ & $0.22 \%$ & $-5.9 \%$ & $0.23 \%$ & $0.23 \%$ & $0.4 \%$ \\
\hline $0.17 \%$ & $0.15 \%$ & $4.6 \%$ & $0.17 \%$ & $0.16 \%$ & $1.9 \%$ & $0.20 \%$ & $0.16 \%$ & $11.2 \%$ \\
\hline $0.11 \%$ & $0.10 \%$ & $3.7 \%$ & $0.11 \%$ & $0.10 \%$ & $4.5 \%$ & $0.10 \%$ & $0.10 \%$ & $-1.1 \%$ \\
\hline
\end{tabular}

\section{Treatment, categorical} data

\begin{tabular}{|c|c|c|c|c|c|c|c|c|c|}
\hline \multicolumn{10}{|l|}{ Before hospitalisation } \\
\hline $\begin{array}{l}\text { Home ventilatory } \\
\text { support }\end{array}$ & $0.02 \%$ & $0.02 \%$ & $-0.3 \%$ & $0.02 \%$ & $0.02 \%$ & $0.8 \%$ & $0.03 \%$ & $0.02 \%$ & $5.9 \%$ \\
\hline $\begin{array}{l}\text { Home oxygen } \\
\text { therapy }\end{array}$ & $0.25 \%$ & $0.29 \%$ & $-9.3 \%$ & $0.25 \%$ & $0.24 \%$ & $0.8 \%$ & $0.29 \%$ & $0.28 \%$ & $2.5 \%$ \\
\hline $\begin{array}{l}\text { Both LAMAs and } \\
\text { LABAs }\end{array}$ & $0.24 \%$ & $0.20 \%$ & $8.3 \%$ & $0.24 \%$ & $0.24 \%$ & $-1.3 \%$ & $0.24 \%$ & $0.22 \%$ & $4.8 \%$ \\
\hline LAMAs only & $0.58 \%$ & $0.67 \%$ & $-17.4 \%$ & $0.58 \%$ & $0.59 \%$ & $-0.6 \%$ & $0.64 \%$ & $0.65 \%$ & $-1.6 \%$ \\
\hline LABAs only & $0.46 \%$ & $0.36 \%$ & $20.7 \%$ & $0.46 \%$ & $0.47 \%$ & $-2.3 \%$ & $0.42 \%$ & $0.40 \%$ & $5.4 \%$ \\
\hline Theophylline & $0.32 \%$ & $0.47 \%$ & $-31.2 \%$ & $0.32 \%$ & $0.33 \%$ & $-0.5 \%$ & $0.45 \%$ & $0.43 \%$ & $4.6 \%$ \\
\hline Expectorants & $0.75 \%$ & $0.76 \%$ & $-1.9 \%$ & $0.75 \%$ & $0.74 \%$ & $1.5 \%$ & $0.71 \%$ & $0.75 \%$ & $-9.2 \%$ \\
\hline $\begin{array}{l}\text { Antibiotic } \\
\text { prescriptions } \\
\text { per } 30 \text { days }\end{array}$ & $0.23 \%$ & $0.31 \%$ & $-11.9 \%$ & $0.23 \%$ & $0.20 \%$ & $5.0 \%$ & $0.26 \%$ & $0.28 \%$ & $-2.8 \%$ \\
\hline $\begin{array}{l}\text { Macrolides per } \\
30 \text { days }\end{array}$ & $0.11 \%$ & $0.19 \%$ & $-19.4 \%$ & $0.11 \%$ & $0.09 \%$ & $8.6 \%$ & $0.14 \%$ & $0.16 \%$ & $-5.2 \%$ \\
\hline
\end{tabular}




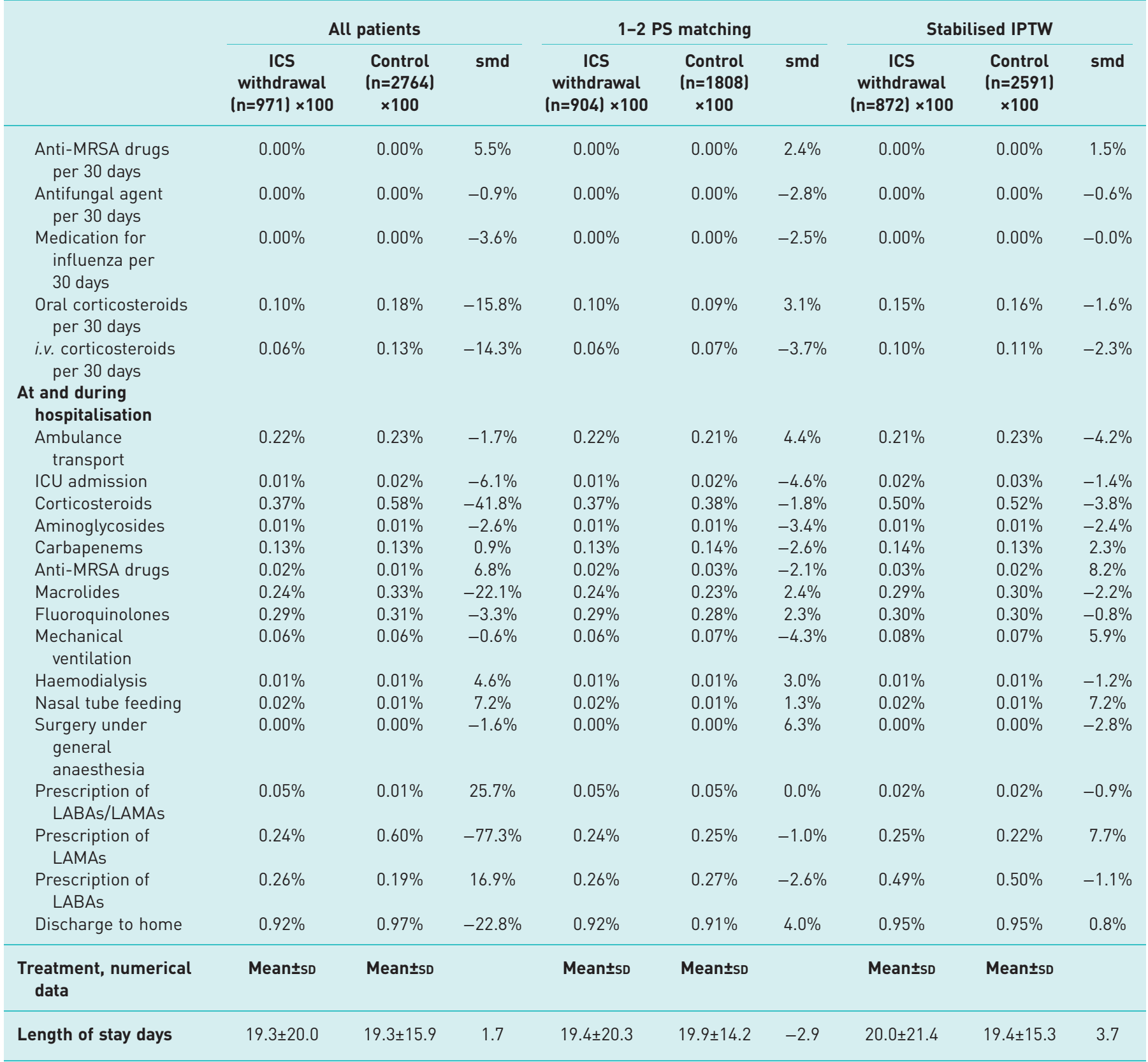

smd: standardised mean difference; GORD: gastro-oesophageal reflux disease; LAMA: long-acting muscarinic antagonist; LABA: long-acting $\beta_{2}$-agonist; SABA: short-acting $\beta_{2}$-agonist; SAMA: short-acting muscarinic antagonist; TMP/SMX: trimethoprim/sulfamethoxazole; MSRA: methicillin-resistant Staphylococcus aureus; ICU: intensive care unit.

were well balanced between the two groups, except for diagnoses of diabetes/abnormal glucose tolerance and constipation or ileus in stabilised IPTW (tables 1 and 2).

The median observation periods after discharge from the first hospitalisation for COPD exacerbation in the ICS withdrawal and control groups were 274 days (IQR, 89-568 days) and 317 days (IQR, 155-692 days) in the PSM population and 343 days (IQR, 107-698 days) and 474 days (IQR, 208-921 days) in the stabilised IPTW population, respectively. The proportions of patients who were re-hospitalised for COPD exacerbation or died within 30 days from discharge were similar in the ICS withdrawal and control groups after 1-2 PSM (4.8\% versus $6.4 \%, \mathrm{p}=0.093)$ and stabilised IPTW (5.9\% versus $5.5 \%, \mathrm{p}=0.826$ ), whereas the proportions within 1 year from discharge were lower in the ICS 

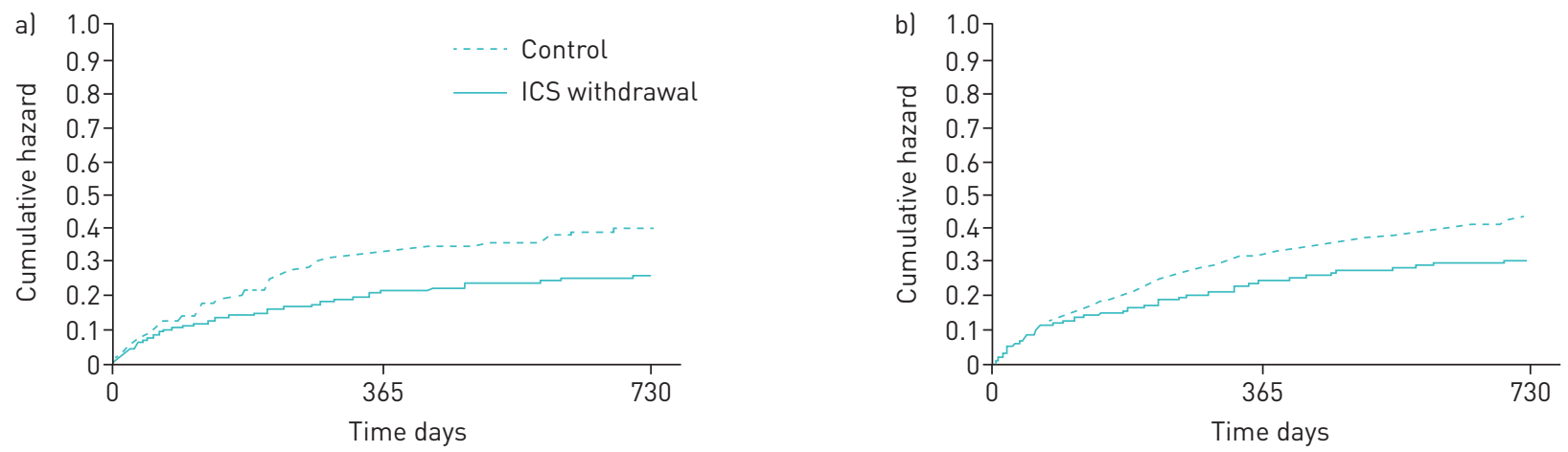

$\begin{array}{lccc}\text { Number at risk } & & & \\ \text { Control } & 1808 & 1320 & 1215 \\ \text { ICS withdrawal } & 904 & 731 & 695\end{array}$

\begin{abstract}
Number at risk
Control 2621

ICS withdrawal 835
\end{abstract}

1895

659
1703

619

FIGURE 2 Cumulative hazard curves for hospitalisation for re-exacerbation or death after hospitalisation for exacerbation in patients with chronic obstructive pulmonary disease (COPD) aged $\geqslant 65$ years with or without inhaled corticosteroid (ICS) withdrawal. Results for a) 1-2 propensity score-matched population; and b) stabilised inverse probability weighted population.

withdrawal group compared to the control group after 1-2 PSM (19.3\% versus 28.4\%, p<0.001) and stabilised IPTW (21.4\% versus $27.9 \%, \mathrm{p}=0.024)$, respectively.

The results of the survival analyses after 1-2 PSM and stabilised IPTW are shown in figure 2 and table 3. Re-hospitalisation or death was significantly reduced in the ICS withdrawal group in both 1-2 PSM and stabilised IPTW. These results were consistent in the subgroup analysis in patients without bronchial asthma, but not in patients with comorbid bronchial asthma (figure S1).

The prescription of antimicrobial medicines in the ICS withdrawal group within 1 year was significantly reduced according to Poisson regression analysis with adjustment for within-hospital clustering after 1-2 PSM and stabilised IPTW, but no significant difference was observed within 30 days. The frequencies of corticosteroid prescriptions were similar in both groups at both 30 days and 1 year (table 4). In the subgroup analyses of patients with and without bronchial asthma, the frequency of antimicrobial prescriptions was significantly higher in the control group within 1 year but not within 30 days after both 1-2 PSM and stabilised IPTW. However, there was no difference in the frequency of corticosteroid prescriptions between the two groups at either time after 1-2 PSM or stabilised IPTW in patients with bronchial asthma (table S3).

The results of the sensitivity analyses are shown in tables S5-S8, and figures S2 and S3. The mean ages of the patients aged $<70$ years after PSM and stabilised IPTW were 63.1 and 62.1 years, respectively. In patients with COPD aged $\geqslant 70$ years, the risks of re-hospitalisation for COPD exacerbation or death were significantly lower in the ICS withdrawal group compared to the control group after both 1-2 PSM and stabilised IPTW, while no differences were observed in patients aged $<70$ years. In patients aged $\geqslant 70$ years, the prescriptions of both antimicrobial medicines and corticosteroids in the ICS withdrawal group within 1 year were significantly reduced after both 1-2 PSM and stabilised IPTW.

TABLE 3 Hazard ratios (HRs) and 95\% confidence intervals of re-hospitalisation for COPD exacerbation or death after 1-2 propensity score (PS) matching and stabilised inverse probability weighting (IPTW) in the inhaled corticosteroid (ICS) withdrawal group versus the control group

\begin{tabular}{llcccc} 
& \multicolumn{4}{c}{ Re-hospitalisation or death } \\
\cline { 2 - 6 } & Study population & ICS withdrawal & Control & HR (95\% CI) & p-value \\
\hline \multirow{2}{*}{ 1-2 PS matching } & All & 904 & 1808 & $0.65(0.52-0.80)$ & $<0.001$ \\
& Comorbid asthma & 330 & 684 & $0.67(0.50-0.91)$ & 0.010 \\
& Without asthma & 574 & 1124 & $0.68(0.51-0.90)$ & 0.008 \\
Stabilised IPTW & All & 835 & 2621 & $0.71(0.56-0.90)$ & 0.005 \\
& Comorbid asthma & 481 & 1513 & $0.82(0.57-1.18)$ & 0.294 \\
& Without asthma & 353 & 1108 & $0.58(0.45-0.75)$ & $<0.001$
\end{tabular}




\begin{tabular}{|c|c|c|c|c|}
\hline & Observation period & Prescription & IRR $(95 \%$ CI) & p-value \\
\hline \multirow[t]{4}{*}{ 1-2 PS matching } & \multirow[t]{2}{*}{30 days } & Antimicrobials & $0.94(0.73-1.23)$ & 0.668 \\
\hline & & Corticosteroids & $0.97(0.72-1.31)$ & 0.856 \\
\hline & \multirow[t]{2}{*}{1 year } & Antimicrobials & $0.78(0.63-0.98)$ & 0.029 \\
\hline & & Corticosteroids & $0.78(0.60-1.02)$ & 0.067 \\
\hline \multirow[t]{4}{*}{ Stabilised IPTW } & \multirow[t]{2}{*}{30 days } & Antimicrobials & $0.90(0.63-1.31)$ & 0.598 \\
\hline & & Corticosteroids & $1.06(0.76-1.47)$ & 0.733 \\
\hline & \multirow[t]{2}{*}{1 year } & Antimicrobials & $0.65(0.51-0.83)$ & 0.001 \\
\hline & & Corticosteroids & $0.76(0.56-1.02)$ & 0.065 \\
\hline
\end{tabular}

\section{Discussion}

This study showed a reduced incidence of re-hospitalisation for COPD exacerbation or death in hospitalised elderly patients with COPD exacerbation following ICS withdrawal, using propensity score analyses. ICS withdrawal was also significantly associated with a decreased frequency of antimicrobial prescriptions in the outpatient setting after discharge, but not with the frequency of systemic corticosteroid prescriptions. However, a marginal difference, albeit not statistically significant, was observed in the frequency of corticosteroid prescriptions within 1 year after discharge in the ICS withdrawal group. Because COPD exacerbation is triggered by lower respiratory tract infection, our findings suggest that ICS withdrawal may also reduce mild-to-moderate COPD exacerbations that do not require re-hospitalisation. The results of the sensitivity analyses supported this notion.

Previous studies investigated ICS withdrawal in patients with COPD at low risk [12, 22-23] and high risk [13] for COPD exacerbation. The current results in elderly patients with COPD were concordant with those previous studies. Accordingly, withdrawal of ICSs in elderly patients with COPD did not increase the risk of re-exacerbation requiring hospitalisation or corticosteroids in the outpatient setting. Similar results were obtained in subgroup analyses in patients with and without bronchial asthma. However, the reduced risk of re-hospitalisation or death after discharge in the ICS withdrawal group among patients with bronchial asthma remained uncertain because of discordance between the results of 1-2 PSM analysis and stabilised IPTW analysis. Nonetheless, ICS withdrawal did not increase the risk of re-hospitalisation or death in either subgroup and may therefore be a viable treatment option in elderly patients with COPD who are hospitalised for exacerbation.

The results for the sensitivity analyses in patients aged $\geqslant 40$ years and $\geqslant 70$ years were similar to those for the primary analyses in patients aged $\geqslant 65$ years; however, no differences between the ICS withdrawal group and control group were observed for patients aged $<70$ years. The mean ages in the primary analyses and sensitivity analyses were approximately 77 and 74 years, respectively. Meanwhile, the mean age of patients aged $<70$ years was approximately 62 years, and comparable to the mean ages in previous studies that showed no difference in COPD exacerbation between patients who continued or discontinued ICSs $[11,13]$. Thus, elderly patients with COPD are more likely to benefit from ICS withdrawal when hospitalised for COPD exacerbation.

The reason for the withdrawal of ICSs in the present study was unknown. However, because the withdrawal decision was made during hospitalisation for COPD exacerbation, ICS withdrawal may have been based on reasonable agreement between the patient and the attending physician, rather than as a consequence of treatment adherence. Adverse drug reactions, including respiratory tract infections, and an inability to continue inhalers may also have been contributory factors.

In this study, patients with COPD who were hospitalised for pneumonia were included among the patients hospitalised for COPD exacerbation. In fact, the proportion of patients with pneumonia at the index hospitalisation was around $80 \%$. The higher incidence of COPD exacerbation in the control group may therefore be explained by the fact that ICS increases the risk of lower respiratory tract infection, including pneumonia, in patients with COPD $[7,8]$, as well as in those with bronchial asthma $[9,10]$. Notably, the mean age of our study population was around 78 years. Elderly patients, especially those with COPD, are known to have a higher risk of lower respiratory tract infection, including pneumonia, and higher pneumonia-related mortality [24-26]. In fact, the frequency of antimicrobial prescriptions during the first 
year was significantly higher in the control group compared to the ICS withdrawal group in our study. The current results, together with those of previous studies, suggest that the higher risk of re-hospitalisation or death after discharge in the control group in our study may have been mainly due to lower respiratory tract infections. Moreover, the higher frequency of antimicrobial prescriptions in the outpatient setting was consistent between patients with COPD with and without bronchial asthma, suggesting similar risks of respiratory tract infections in patients with COPD and ACO.

Some patients in our study withdrew bronchodilators as well as ICSs during hospitalisation for COPD exacerbation. Bronchodilators are reportedly effective for preventing exacerbation in patients with COPD [1]. Moreover, the elderly patients who became incapable of continuing both ICSs and bronchodilators were presumably those with the lowest levels of physical activity who were unable to keep using inhaler devices. Such patients with poor physical activity are reportedly susceptible to COPD exacerbation and pneumonia [1]. In this study, we adjusted for prescriptions of bronchodilators during hospitalisation, together with the Hugh-Jones dyspnoea score at admission and activities of daily living scores at discharge, which were significantly imbalanced before adjustment. It is therefore highly unlikely that the current results could be explained by the difference in the proportions of patients who continued bronchodilators between the two groups.

Although the role of ICSs in patients with asthma has been established, treatment recommendations for ACO, including the use of ICSs, are mainly extrapolated from studies of patients with bronchial asthma or COPD [4]. Interestingly, although ICSs are recommended for ACO, our study showed no association between ICS withdrawal in elderly patients with COPD and comorbid bronchial asthma and re-exacerbation of COPD or death or the frequency of oral corticosteroid prescriptions at either 30 days or 1 year after discharge in the outpatient setting. This suggests that neither asthma attacks requiring hospitalisation nor systemic corticosteroids may have increased after ICS withdrawal in this population. Furthermore, the frequency of antimicrobial medicine prescriptions was significantly higher at 1 year in both analyses with 1-2 PSM and stabilised IPTW. These results suggest that withdrawal of ICSs may benefit even elderly patients with ACO after hospitalisation for COPD exacerbation. Presumably, the risk-benefit balance of ICS may alter in elderly patients with COPD and ACO. Further studies evaluating the risks and benefits of ICSs are therefore required, particularly in elderly patients.

Previous studies have demonstrated the existence of a patient population that deteriorates following ICS withdrawal. This includes the post hoc analysis of the aforementioned study that evaluated ICS withdrawal in patients with COPD after long-term triple therapy [27]. Patients with $\geqslant 300$ blood eosinophils $\mu \mathrm{L}^{-1}$ had a higher risk of exacerbation after ICS withdrawal [11, 27]. However, a recent database study that evaluated the association between eosinophil counts and COPD exacerbation or all-cause mortality reported that ICS withdrawal did not increase the risk, even in patients with eosinophilia [28]. Because our study only included patients who were discharged before March 2016, it is unlikely that recent studies referring to the role of blood eosinophil counts induced confounding by indication.

Because asthma is known to be a heterogeneous disease in terms of its phenotype and severity [29], patients with COPD and comorbid asthma are presumably also heterogeneous. It is therefore likely that some elderly patients with ACO will require ICSs even after hospitalisation for COPD exacerbation.

This study had some limitations. Firstly, the diagnoses in the database may have been less well validated than those in planned prospective studies. However, diagnosis of chronic pulmonary disease was reported to have a sensitivity of $33.3 \%$ and a specificity of $96.9 \%$ in the Diagnosis Procedure Combination database [30]. Secondly, data for patients who visited hospitals other than their discharged hospital or had out-of-hospital death could not be collected. Thirdly, we were not able to take account of the effects of vaccinations (i.e. anti-influenza vaccines or pneumococcal vaccines) due to a lack of data. Fourthly, we could not evaluate the severity of bronchial asthma and COPD, blood eosinophil counts, and pulmonary function tests because of a lack of data. Fifthly, because the Diagnosis Procedure Combination database only contains data for date of ICS prescription, and not data for the actual date of ICS use, the process of ICS withdrawal could not be evaluated. Finally, this was a retrospective observational study and causality could therefore not be established.

\section{Conclusion}

The present results suggest that withdrawal of ICSs may reduce the risk of re-hospitalisation for COPD exacerbation or death among elderly patients with COPD in an outpatient setting, associated with a reduced frequency of antimicrobial prescriptions and no increased frequency of systemic corticosteroid prescriptions. We therefore conclude that ICS withdrawal is safe in elderly patients with COPD who are hospitalised for exacerbation. Further studies are needed to specify the patient population among elderly patients with COPD who may benefit from ICS discontinuation.

Author contributions: T. Jo designed the study, analysed and interpreted the data, and prepared the manuscript. H. Yasunaga analysed and interpreted the data, and prepared the manuscript. Y. Yamauchi designed the study, analysed and interpreted 
the data. A. Mitani analysed and interpreted the data. Y. Hiraishi analysed and interpreted the data. W. Hadegawa designed the study and interpreted the data. Y. Sakamoto designed the study and interpreted the data. H. Matsui collected and interpreted the data. K. Fushimi collected and interpreted the data. T. Nagase designed the study, interpreted the data and prepared the manuscript. All authors approved the final manuscript.

Support statement: This work was supported by grants from the Ministry of Health, Labour and Welfare, Japan (19AA2007 and H30-Policy-Designated-004) and the Ministry of Education, Culture, Sports, Science and Technology, Japan (17H04141). The funding bodies had no role in the design of the study; collection, analysis, or interpretation of the data; or writing of the manuscript.

Conflict of interest: T. Jo has nothing to disclose. H. Yasunaga reports grants from Ministry of Health, Labour, and Welfare, Japan, during the conduct of the study. Y. Yamauchi has nothing to disclose. A. Mitani has nothing to disclose. Y. Hiraishi has nothing to disclose. W. Hasegawa has nothing to disclose. Y. Sakamoto has nothing to disclose.

H. Matsui has nothing to disclose. K. Fushimi has nothing to disclose. T. Nagase has nothing to disclose.

\section{References}

1 Global Initiative for Chronic Obstructive Lung Disease (GOLD); 2019 Report. https://goldcopd.org/ Date last accessed: August 2, 2019. Date last updated: 2019.

2 Martinez FJ, Vestbo J, Anderson JA, et al. Effect of fluticasone furoate and vilanterol on exacerbations of chronic obstructive pulmonary disease in patients with moderate airflow obstruction. Am J Respir Crit Care Med 2017; 195: 881-888.

3 Yang IA, Shaw JG, Goddard JR, et al. Use of inhaled corticosteroids in COPD: improving efficacy. Expert Rev Respir Med 2016; 10: 339-350.

4 A joint project of GINA and GOLD: asthma, COPD, and asthma-COPD overlap syndrome (ACOS). https://goldcopd. org/asthma-copd-asthma-copd-overlap-syndrome/ Date last accessed: August 2, 2019. Date last updated: 2015.

5 Pedros C, Formiga F, Corbella X, et al. Adverse drug reactions leading to urgent hospital admission in an elderly population: prevalence and main features. Eur J Clin Pharmacol 2016; 72: 219-226.

6 Battaglia S, Cardillo I, Lavorini F, et al. Erratum to: safety considerations of inhaled corticosteroids in the elderly. Drugs Aging 2015; 32: 1067-1076.

7 Eurich DT, Lee C, Marrie TJ, et al. Inhaled corticosteroids and risk of recurrent pneumonia: a population-based, nested case-control study. Clin Infect Dis 2013; 57: 1138-1144.

8 Cascini S, Kirchmayer U, Belleudi V, et al. Inhaled corticosteroid use in chronic obstructive pulmonary disease and risk of pneumonia: a nested case-control population-based study in Lazio (Italy)-The OUTPUL Study. COPD 2017; 14: 311-317.

9 Qian CJ, Coulombe J, Suissa S, et al. Pneumonia risk in asthma patients using inhaled corticosteroids: a quasi-cohort study. Br J Clin Pharmacol 2017; 83: 2077-2086.

10 Hojo M, Iikura M, Hirano S, et al. Increased risk of nontuberculous mycobacterial infection in asthmatic patients using long-term inhaled corticosteroid therapy. Respirology 2012; 17: 185-190.

11 Chapman KR, Hurst JR, Frent SM, et al. Long-term triple therapy de-escalation to indacaterol/glycopyrronium in COPD patients (SUNSET): a randomized, double-blind, triple-dummy clinical trial. Am J Respir Crit Care Med 2018; 198: 329-339.

12 Vogelmeier C, Worth H, Buhl R, et al. 'Real-life' inhaled corticosteroid withdrawal in COPD: a subgroup analysis of DACCORD. Int J Chron Obstruct Pulmon Dis 2017; 12: 487-494.

13 Magnussen H, Disse B, Rodriguez-Roisin R, et al. Withdrawal of inhaled glucocorticoids and exacerbations of COPD. N Engl J Med 2014; 371: 1285-1294.

14 Calzetta L, Matera MG, Braido F, et al. Withdrawal of inhaled corticosteroids in COPD: A meta-analysis. Pulm Pharmacol Ther 2017; 45: 148-158.

15 Yasunaga $\mathrm{H}$, Matsui $\mathrm{H}$, Horiguchi $\mathrm{H}$, et al. Health services research using the diagnosis procedure combination database in Japan. Asian Pac J Dis Manag 2013; 7: 19-24.

16 Mahoney FI, Barthel DW. Functional evaluation: the Barthel Index. Md State Med J 1965; 14: 61-65.

17 Japan Meteorological Agency. General Information on Climate of Japan. www.data.jma.go.jp/gmd/cpd/longfcst/en/ tourist.html Date last accessed: August 2, 2019.

18 Rosenbaum PR, Rubin DB. Constructing a control group using multivariate matched sampling methods that incorporate the propensity score. Am Stat 1985; 39: 33-38.

19 Xu S, Ross C, Raebel MA, et al. Use of stabilized inverse propensity score as weight to directly estimate relative risk and its confidence intervals. Value Health 2010; 2: 273-237.

20 Austin PC. Using the standardized difference to compare the prevalence of a binary variable between two groups in observational research. Commun Stat Simul Comput 2009; 38: 1228-1234.

21 Williams RL. A note on robust variance estimation for cluster-correlated data. Biometrics 2000; 56: 645-646.

22 Rossi A, Guerriero M, Corrado A, et al. Withdrawal of inhaled corticosteroids can be safe in COPD patients at low risk of exacerbation: a real-life study on the appropriateness of treatment in moderate COPD patients (OPTIMO). Respir Res 2014; 15: 77.

23 Rossi A, van der Molen T, del Olmo R, et al. INSTEAD: a randomised switch trial of indacaterol versus salmeterol/fluticasone in moderate COPD. Eur Respir J 2014; 44: 1548-1556.

24 Jackson ML, Neuzil KM, Thompson WW, et al. The burden of community-acquired pneumonia in seniors: results of a population-based study. Clin Infect Dis 2004; 39: 1642-1650.

25 Sligl WI, Eurich DT, Marrie TJ, et al. Age still matters: prognosticating short- and long-term mortality for critically ill patients with pneumonia. Crit Care Med 2010; 38: 2126-2132.

26 Faverio P, Aliberti S, Bellelli G, et al. The management of community-acquired pneumonia in the elderly. Eur J Intern Med 2014; 25: 312-319.

27 Watz H, Tetzlaff K, Wouters EF, et al. Blood eosinophil count and exacerbations in severe chronic obstructive pulmonary disease after withdrawal of inhaled corticosteroids: a post hoc analysis of the WISDOM trial. Lancet Respir Med 2016; 4: 390-398. 
28 Oshagbemi OA, Franssen FME, van Kraaij S, et al. Blood eosinophil counts, withdrawal of inhaled corticosteroids and risk of COPD exacerbations and mortality in the clinical practice research datalink (CPRD). COPD 2019; 16 : 152-159.

29 Perez-de-Llano L. Cosio BG on behalf of the CHACOS Study Group. Asthma-COPD overlap is not a homogeneous disorder: further supporting data. Respir Res 2017; 18: 183.

30 Yamana H, Moriwaki M, Horiguchi H, et al. Validity of diagnoses, procedures, and laboratory data in Japanese administrative data. J Epidemiol 2017; 27: 476-482. 